\title{
Parámetros bioquímicos sanguíneos en llamas (Lama glama) alimentadas en praderas nativas tholar pajonal en Choquecota Oruro, Bolivia
}

\author{
Blodd biochemistry parameters in llamas (Lama glama) grassed in native prairie tholar \\ pajonal in Choquecota Oruro, Bolivia \\ Parâmetros bioquímicos sanguíneos em lhamas (Lama glama) alimentados em pradarias \\ nativas em Choquecota Oruro, Bolívia
}

\author{
Santiago Copa \\ copasanty@gmail.com \\ Rene Condori \\ rcondoriq_25@hotmail.com
}

Instituto de Investigaciones en Ciencia Animal y Post Grado (ICA) Carrera Ingeniería en Zootecnia e Industria Pecuaria, Universidad Pública de El Alto, Bolivia

Artículo recibido actubre 2019, arbitrado noviembre 2019 y publicado en enero 2020

\section{RESUMEN}

Con el objetivo de determinar los parámetros bioquímicos sanguíneos en llamas alimentadas en praderas naturales tholar pajonal, se tomó muestras de sangre por punción yugular de 62 llamas distribuidas por categorías de edad, que fueron procesadas en analizador de química sanguínea VetScanVS2 utilizando el Regent Rotor ABAXIS, los datos se analizaron con un diseño completamente al azar y las diferencias estadísticas por la prueba de comparación de medias de Duncan. Los resultados fueron: proteína total (PT) 6,60+0,51 g/dL, albúmina (ALB) 4,29+0,36 g/dL, globulina (GLOB) $2,29+0,55 \mathrm{~g} / \mathrm{dL}$, urea nitrógeno (BUN) 6,89+4,60 $\mathrm{mg} / \mathrm{dL}$, fosfatasa alcalina (ALP) 87,39+47,78 UI/L, alanina aminotransferasa (ALT) 10,42+3,60 UI/L, amilasa (AMY) 671,61+124,06 UI/L, creatinina (CRE) 2,52+0,49 mg/dL, bilirrubina total (TBIL) $0,30 \mathrm{mg} / \mathrm{dL}$, glucosa (GLU) 105,65+17,83 mg/dL, calcio (CA) 9,30+0,33 mg/dL, fósforo (PHOS) 9,09+1,19 mg/dL, sodio (NA) 147,08+3,04 mEq/L y potasio (K) 5,99+0,80 $\mathrm{mEq} / \mathrm{L}$. La edad de llamas influye sobre los parámetros sanguíneos $(P \leq 0,01)$ de PT (proteína total), ALB, GLOB, BUN, ALP, AMY, CRE, GLU, CA, PHOS; mientras la ALT,TBILL,NA y K no están influidos por la edad $(P>0,05)$. En llamas cada individuo posee patrones diferentes de componentes químicos sanguíneos que pueden cambiar con la edad.

Palabras clave: Llamas; bioquímicos; parámetros, sanguíneos

\begin{abstract}
With the objective of determining the blod biochemistry parameters in llamas grassed in native prairie tholar pajonal, took blood samples from the yugular vein of 62 llamas distributed by age categories, that fueton processed in blood chemistry analyser VetScanVS2 using the Regent Rotor ABAXIS, the data were analyzed using a completely randomized desing and statistical differences for the mean comparison test of Duncan. The results of: protein total (TP) $6,60+0,51 \mathrm{~g} / \mathrm{dL}$, albumin (ALB) 4,29+0,36 g/dL, globulin (GLOB) $2,29+0,55 \mathrm{~g} / \mathrm{dL}$, urea nitrogen (BUN) 6,89+4,60 $\mathrm{mg} / \mathrm{dL}$, alkaline phosphatase (ALP) $87,39+47,78$ UI/L, alanine aminotransferase (ALT) 10,42+3,60 UI/L, amylase (AMY) 671,61+124,06 UI/L, creatinine (CRE) 2,52+0,49 mg/dL, total bilirrubin (TBIL) $0,30 \mathrm{mg} / \mathrm{dL}$, glucose (GLU) $105,65+17,83$ $\mathrm{mg} / \mathrm{dL}$, calcium (CA) 9,30+0,33 mg/dL, phosphorus (PHOS) 9,09+1,19 $\mathrm{mg} / \mathrm{dL}$, sodium (NA) $147,08+3,04 \mathrm{mEq} / \mathrm{L}$ y potassium (K) 5,99+0,80 $\mathrm{mEq} / \mathrm{L}$. the age of flames influence blood parameters $(P \leq 0,01)$ de TP (total protein), ALB, GLOB, BUN, ALP, AMY, CRE, GLU, CA, PHOS; while the ALT,TBILL,NA y $K$ they are not influenced by age $(\mathrm{P}>0,05)$. In llamas every individual possesses chemical patterns of blood components that can change with age.
\end{abstract}

Key words: Llamas; biochemistry; parameters, blood 


\section{RESUMO}

Para determinar os parâmetros bioquímicos do sangue em chamas alimentadas em pradarias naturais do tolar, foram coletadas amostras de sangue por punção jugular de 62 chamas distribuídas por categorias de idade, que foram processadas em um analisador químico de sangue VetScanVS2 usando o Regent Rotor ABAXIS, os dados foram analisados com delineamento inteiramente casualizado e diferenças estatísticas pelo teste de comparação de médias de Duncan. Os resultados foram: proteína total (PT) 6,60 + 0,51 g / dL, albumina (ALB) 4,29+0,36 g / dL, globulina (GLOB) 2,29+0,55 g / dL, nitrogênio uréia (BUN) $6,89+4,60 \mathrm{mg} / \mathrm{dL}$, fosfatase alcalina (ALP) $87,39+47,78$ UI / L, alanina aminotransferase (ALT) 10,42 + 3,60 UI / L, amilase ( AMY) 671,61 + 124,06 UI / L, creatinina (CRE) $2,52+0,49 \mathrm{mg} / \mathrm{dL}$, bilirrubina total (TBIL) $0,30 \mathrm{mg} / \mathrm{dL}$, glicose (GLU) 105,65 + 17, $83 \mathrm{mg} / \mathrm{dL}$, cálcio (CA) 9,30 + 0,33 mg / dL, fósforo (PHOS) 9,09 + 1,19 mg / dL, sódio (NA) 147,08 + 3,04 mEq / L e potássio (K) 5,99+0,80 mEq / L. A idade dos lhamas influencia os parâmetros sanguíneos $(\mathrm{P} \leq 0,01)$ do $\mathrm{TP}$ (proteína total), ALB, GLOB, BUN, ALP, AMY, CRE, GLU, CA, PHOS; enquanto ALT, TBILL, NA e K não são influenciados pela idade $(\mathrm{P}>0,05)$. Em chamas, cada indivíduo tem padrões diferentes de componentes químicos do sangue que podem mudar com a idade.

Palavras-chave: Lhamas; bioquímicos; parâmetros, sangrento

\section{INTRODUCCIÓN}

En la comunidad altiplánica de Choquecota, las familias campesinas crían las llamas bajo un sistema extensivo en praderas nativas tholar y pajonal, donde se observa la época más crítica los meses de mayo a noviembre, por la disponibilidad de pastos de baja calidad nutritiva debido a las condiciones climáticas adversas (heladas y sequía); factor que determina los bajos rendimientos productivos y reproductivos de estos animales.

El crecimiento y la nutrición de llamas se afectan mutuamente, existe poca información sobre los niveles bioquímicos sanguíneos en llamas en su hábitat natural. Las estrategias para mejorar la producción y la productividad de la llama están orientadas a mejorar la nutrición, con el uso de las pruebas de química sanguínea es posible diferenciar entre una enfermedad predominantemente hepatocelular o colestática, evaluar la severidad y cronicidad de una condición de salud y nutrición (Scholz, 2001).

Los objetivos del presente trabajo fueron: Determinar los parámetros bioquímicos sanguíneos (PT, ALB, GLOB, BUN, ALP, ALT, AMY, CRE, TBILL, GLU, CA, PHOS, NA y K) en llamas alimentadas en praderas nativas tholar pajonal según edades, en Choquecota, provincia Carangas del departamento de Oruro, Bolivia.

\section{MATERIALES Y MÉTODOS}

El presente trabajo de investigación se realizó en la comunidad Lerco, segunda sección municipal Choquecota, provincia Carangas del departamento Oruro, Bolivia, entre 16007'94" de Latitud Sur y 683' $64^{\prime \prime} 17^{\circ} 35^{\prime}$ Longitud Oeste y a 3.868 msnm. La zona presenta una precipitación pluvial media anual que varía entre 300 a 400 $\mathrm{mm}$, la temperatura media anual es $3.4^{\circ} \mathrm{C}$ con una máxima de $17.2^{\circ} \mathrm{C}$ y una mínima de $-12^{\circ} \mathrm{C}$.

Las familias de la comunidad Lerco se caracterizan por su vocación productiva de llamas de carne ecológica, estos animales son pastoreados en praderas naturales tholares y pajonales que son la principal vegetación de la región.

El presente estudio se ha realizado con llamas en su habitad natural. El muestreo fue no probabilístico, con un total de 62 llamas 
distribuidas en cuatro categorías de edad: 15 animales dientes de leche (DL), 15 animales dos dientes (DD), 15 animales cuatro dientes (CD) y 17 animales boca llena (BLl); que proceden de dos tamas (rebaños) de familias campesinas productoras de llamas.

Se obtuvo muestras de sangre por punción en la vena yugular en tubos de $2 \mathrm{~mL}$ con heparina debidamente identificados. Utilizando una micropipeta se llenaron 100 $\mu \mathrm{L}$ de sangre en el Regent Rotor ABAXIS que luego fue procesada en el analizador de química sanguínea VetScanVS2 con el que fueron determinadas los parámetros de proteína total (PT), albúmina (ALB), globulina (GLOB), urea nitrógeno (BUN), fosfatasa alcalina (ALP), alanina aminotransferasa (ALT), amilasa (AMY), creatinina (CRE), bilirrubina total (TBIL), glucosa (GLU), calcio (CA), fósforo (PHOS), sodio (NA) y potasio (K).

El tipo de investigación es explicativo, los resultados fueron analizados con el diseño completamente al azar y las diferencias mediante la prueba de comparación de medias de Duncan (? =0.05). Se han verificado los supuestos de normalidad y homogeneidad de varianzas.

\section{RESULTADOS Y DISCUSIÓN}

\section{Parámetros químicos sanguíneos en Llamas}

La categoría de edad de llamas influye sobre los parámetros sanguíneos $(\mathrm{P} \leq 0,01)$ de proteína total, albúmina, globulina, urea nitrógeno, creatinina, glucosa, calcio, fósforo y la actividad enzimática de fosfatasa alcalina y amilasa; mientras la bilirrubina total, sodio, potasio y la actividad enzimática alanina aminotransferasa, no están influidos por la edad $(\mathrm{P}>0,05)$. (Tabla 1$)$.

Los resultados de parámetros bioquímicos sanguíneos en llamas, están dentro los valores reportados por investigaciones anteriores; Coaquira (2005), Ajata (2006), Quispe (2007), Ali (2008), Choque (2009), Fowler (2010); excepto el sodio y urea nitrógeno que son ligeramente inferiores y para amilasa no existen parámetros.

Tabla 1. Parámetros bioquímicos sanguíneos en llamas según categorías de edad

\begin{tabular}{|c|c|c|c|c|c|c|c|c|c|c|c|c|c|c|}
\hline Variable & $\begin{array}{c}\text { TP } \\
\mathrm{g} / \mathrm{dL}\end{array}$ & $\begin{array}{l}\text { ALB } \\
\text { g/dL }\end{array}$ & $\begin{array}{l}\text { GLOB } \\
\text { g/dL }\end{array}$ & $\begin{array}{c}\mathrm{BUN} \\
\mathrm{mg} / \mathrm{dL}\end{array}$ & $\begin{array}{c}\text { ALP } \\
\text { UI/L }\end{array}$ & $\begin{array}{l}\text { ALT } \\
\text { UI/L }\end{array}$ & $\begin{array}{l}\text { AMY } \\
\text { UI/L }\end{array}$ & $\begin{array}{c}\text { CRE } \\
\mathrm{mg} / \mathrm{dL}\end{array}$ & $\begin{array}{c}\text { TBIL } \\
\mathrm{mg} / \mathrm{dL}\end{array}$ & $\begin{array}{c}\text { GLU } \\
\mathrm{mg} / \mathrm{dL}\end{array}$ & $\begin{array}{c}\mathrm{CA} \\
\mathrm{mg} / \mathrm{dL}\end{array}$ & $\begin{array}{r}\text { PHOS } \\
\text { L mg/dL }\end{array}$ & $\begin{array}{c}\mathrm{NA} \\
\mathrm{mEq} / \mathrm{L}\end{array}$ & $\begin{array}{c}\mathrm{K} \\
\mathrm{mEq} / \mathrm{L}\end{array}$ \\
\hline & 27 & 4,45 & 1,83 & 12,33 & 147,40 & & & 2,73 & 0,30 & 113,67 & 9,45 & 10,01 & 146,93 & 5,89 \\
\hline & 6,75 & 4,27 & 2,49 & 4,53 & 74,27 & 9,53 & & 2,34 & 0,30 & 108,67 & 9,27 & 9,11 & 147,33 & 6,17 \\
\hline $\begin{array}{l}\text { Cuatro } \\
\text { dientes }\end{array}$ & 6,83 & 4,35 & 2,34 & 4,80 & 60,07 & 11,27 & 660,53 & 2,53 & 0,30 & 99,40 & 9,31 & 8,91 & 147,40 & 5,74 \\
\hline & 6,53 & 4,09 & 2,46 & 6,00 & 70,12 & 10,35 & 635,12 & 2,47 & 0,30 & 101,41 & 9,18 & 8,42 & 146,71 & 6,15 \\
\hline Promedio & 6,60 & 4,29 & 2,29 & 6,89 & 87,39 & 10,42 & 671,61 & 2,52 & 0,30 & 105,65 & 9,30 & 9,09 & 147,08 & 5,99 \\
\hline
\end{tabular}




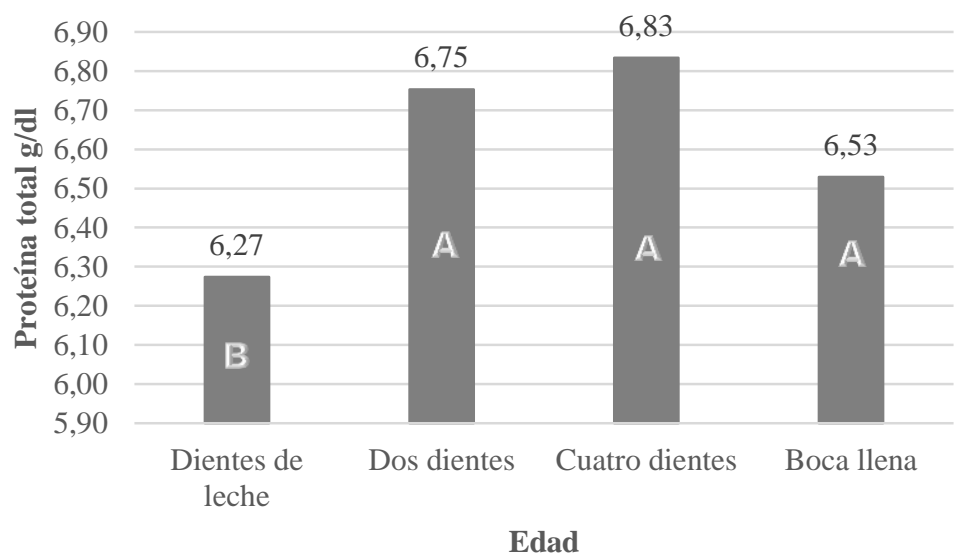

Gráfico 1. Parámetros sanguíneos de proteína total en llamas

Las proteínas son los principales constituyentes del cuerpo animal y esencial para la reparación celular y procesos de síntesis. Las llamas DD, CD, BLl, presentan niveles sanguíneos similares de PT y superiores a los animales DL $($ 团 $=0.05)$. (Gráfico 1).

El presente estudio mostró los menores niveles sanguíneos de PT en llamas DL, probablemente se deban a que estos animales por su estado fisiológico utilizan más proteínas para el crecimiento tisular $\mathrm{y}$ corporal. Las diferencias de niveles sanguíneos de PT por categoría de edad están influidas por el estado fisiológico de los animales (crecimiento) y la calidad nutritiva de los pastos nativos que a su vez están relacionadas con las características climáticas en los meses del año.

El tipo de ración afecta la digestibilidad de proteína de las raciones (Choque 2009). Los microorganismos del rumen tiene un efecto nivelador sobre el aporte de proteína al animal; suplementan cualitativamente $\mathrm{y}$ cuantitativamente las proteínas de los alimentos groseros de mala calidad (Donald, 1999).

\section{Parámetros sanguíneos de albúmina en llamas}

La albúmina es el responsable del $80 \%$ de la presión osmótica coloidal total que mantiene el movimiento de los líquidos del cuerpo, facilita el metabolismo y desintoxicación de diversas sustancias y potencia la eliminación de los radicales libres del plasma. Los niveles sanguíneos de ALB son superiores en llamas DL e inferiores en llamas BLl, mientras que las llamas DD y CD presentan niveles de ALB similares a los animales DL y BLl. (圆=0,05). (Gráfico 2).

Las llamas dientes de leche, por la intensa actividad metabólica debido al crecimiento corporal, tienen niveles de albumina sanguínea superiores para el transporte de diferentes compuestos necesarios para el desarrollo del animal. En llamas adultas las funciones vitales tienden a disminuir aspecto que influye para que las concentraciones sanguíneas de albumina disminuyan. Para Radostits et al. (2002) las concentraciones de albúmina disminuyen a medida que aumenta la edad del animal. 


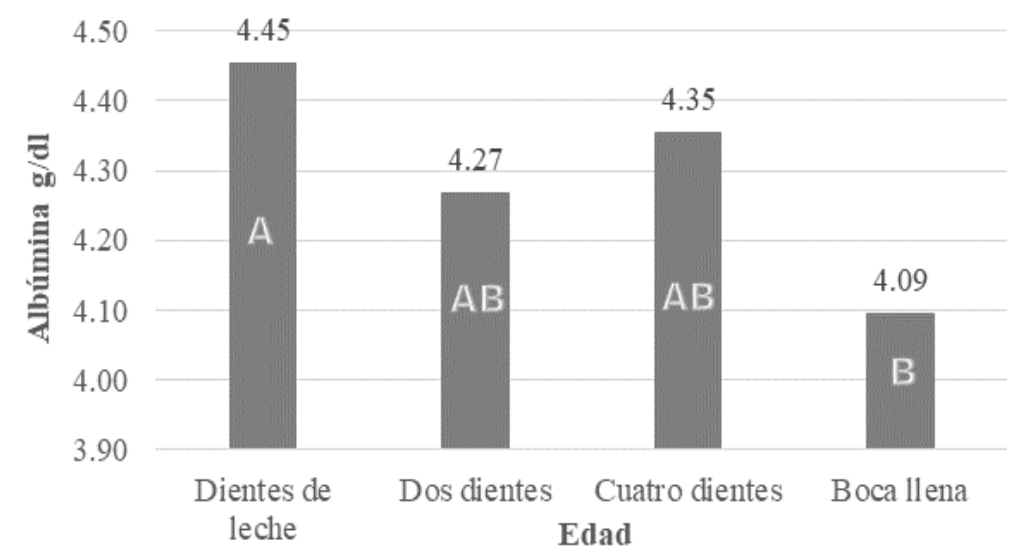

Gráfico 2. Parámetros sanguíneos de albúmina en llamas

\section{Parámetros sanguíneos de globulina en llamas}

Las globulinas (Gráfico 3) son los constituyentes fundamentales de los anticuerpos, pueden actuar como proteínas transportadoras que vinculan sustancias específicas. Los parámetros de globulinas son similares en llamas DD, CD, BLl y superiores a DL (

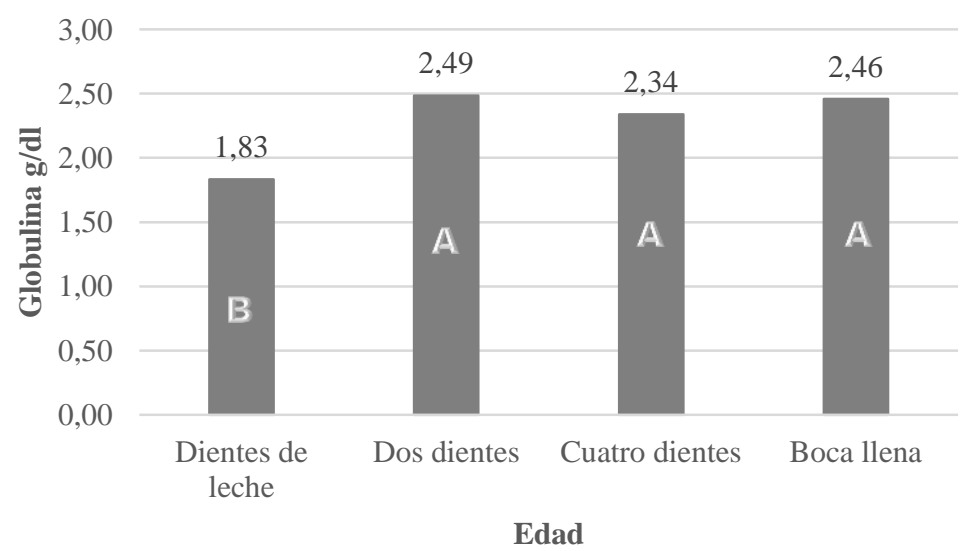

Gráfico 3. Parámetros sanguíneos de globulina en llamas

Los menores niveles sanguíneos de globulina en llamas DL, probablemente se deban a que utilizan para el crecimiento tisular y corporal. Los niveles superiores de globulina en los animales de DD, CD y BLl se deben a que estos animales tienen mayor capacidad digestiva. Las diferencias en los niveles sanguíneos de GLOB por edad están influidas por el estado fisiológico e inmunológico de los animales y la calidad nutritiva de los pastos. (Gráfico 3).
Las bajas concentraciones sanguíneas de globulinas denotan disproteinemia, por posibles problemas carenciales (Murray, et al., 2007). La hiperglobunemia se asocia con la respuesta humoral reciente a infecciones o vacunación mientras que la hipoglobunemia puede indicar una baja respuesta o un deficiente estado inmunológico (Álvarez, 2008). 
Parámetros sanguíneos de urea calidad nutritiva que favorecen el mayor

\section{nitrógeno en llamas}

El hígado de las llamas cumple un papel clave en el metabolismo y formación de la urea a partir del amonio absorbido por las paredes de los C1 y C2, aminoácidos y péptidos absorbidos a nivel duodenal. Los niveles de urea son superiores en llamas DL e inferiores en llamas de DD, CD, BLl (? $=0.05$ ) que son similares entre sí.

Los animales jóvenes dientes leche tienen capacidad selectiva de pastos de buena desarrollo de microorganismos en los compartimientos C1 y C2 por lo que los niveles sanguíneos de urea son superiores. En los animales de mayor edad los niveles inferiores indican que la ingesta proteica es mínima debido a la baja calidad de los pastos y mayor requerimiento de proteínas por su estado fisiológico reproductivo. (Gráfico 4).

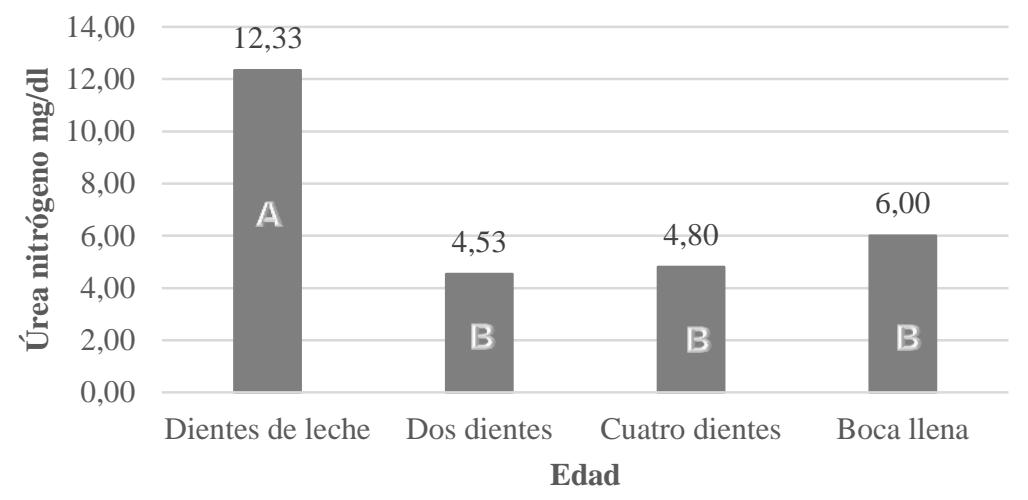

Gráfico 4. Parámetros sanguíneos de urea nitrógeno en llamas

Cuando existe menor concentración de urea en el plasma sanguíneo del animal puede ocurrir por la baja ingestión de proteínas como por la mala absorción de proteína o sobre hidratación del animal (Benjamín, 1991).

\section{Parámetros sanguíneos de fosfatasa alcalina en llamas}

La fosfatasa alcalina corresponde a un grupo de enzimas que intervienen en la hidrólisis de las uniones éster del ácido ortofosfórico a pH alcalino, las fuentes más importantes son el hígado, los huesos y el intestino. La actividad enzimática de ALP son superiores en llamas DL e inferiores en DD, CD, BLl que son similares entre sí (

Las llamas dientes de leche por el aumento de la fracción ósea que traduce la actividad osteoblástica del hueso (crecimiento) presentan elevación fisiológica de la actividad enzimática de fosfatasa alcalina; situación que disminuye en los animales de mayor edad. (Gráfico 5). 


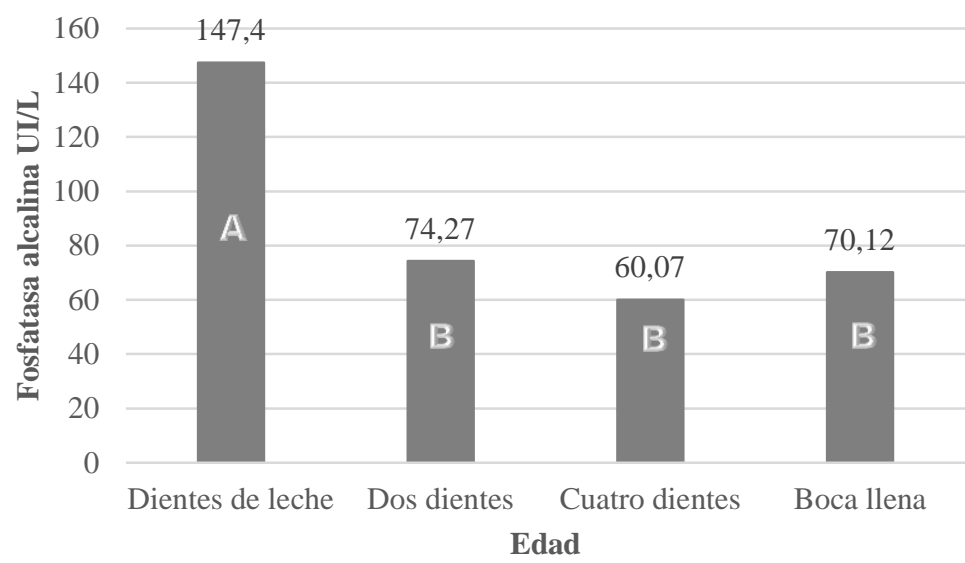

Gráfico 5. Parámetros sanguíneos de fosfatasa alcalina en llamas

\section{Parámetros sanguíneos de amilasa en llamas}

La alanina aminotransferasa son enzimas que transfieren un aminoácido a un cetoácido aceptor para dar lugar a aminoácidos distintos de los originales. La actividad enzimática de la alanina aminotransferasa en las diferentes edades de llamas no presenta diferencias estadísticas. Los valores obtenidos están dentro los parámetros reportados por diferentes investigadores.
La amilasa es una enzima que se origina en el páncreas y glándulas salivales. La actividad enzimática de amilasa son superiores en llamas DD e inferiores en BLl, y en llamas DL, CD son similares a las anteriores $\quad($ Q $=0.05)$. Las diferencias corresponden a variaciones fisiológicas de los animales. (Gráfico 6).

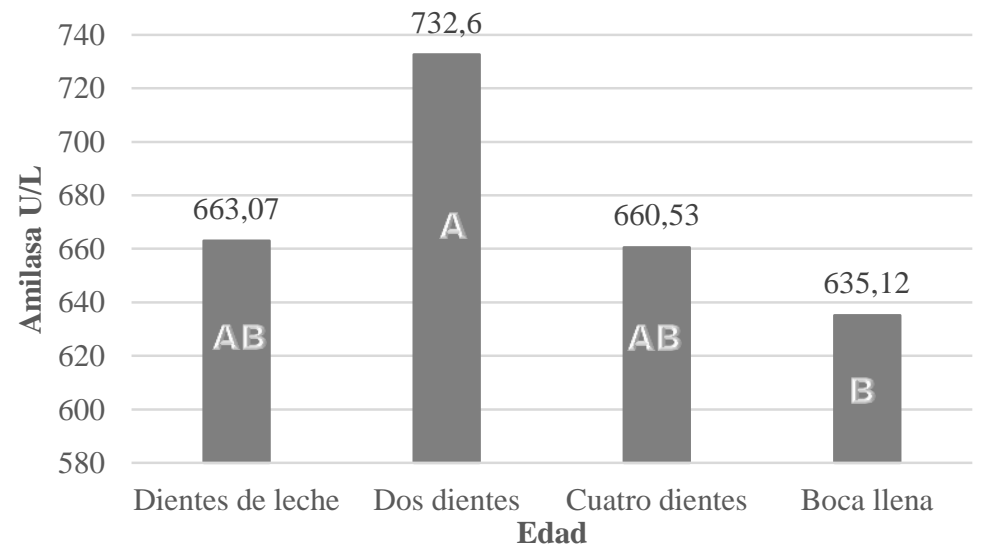

Gráfico 6. Parámetros sanguíneos de amilasa en llamas

Parámetros sanguíneos de creatinina en llamas

La creatinina es el producto resultante del catabolismo muscular, las concentraciones sanguíneas se relacionan con el volumen de filtrado glomerular. Los niveles de creatinina sanguíneos son superiores en llamas DL e inferiores en DD, en llamas CD y BLl son similares a las anteriores ( $(2=0.05)$. 
Los niveles superiores en llamas DL se relacionan con el estado fisiológico de crecimiento, en los animales de mayor edad disminuyen estos niveles por el envejecimiento; en llamas de DD los niveles son inferiores por la menor masa muscular.

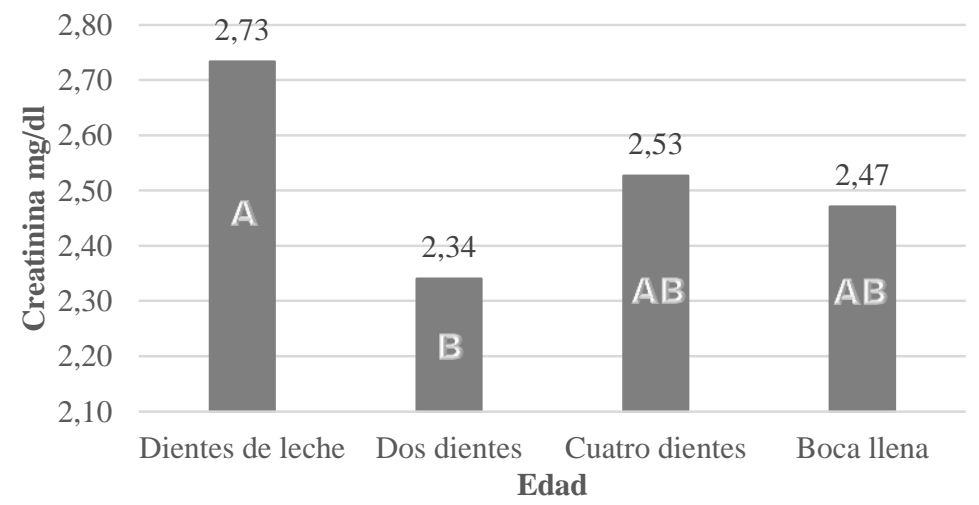

Gráfico 7. Parámetros sanguíneos de creatinina en llamas

La creatinina solo se encuentra en mayor cantidad en animales en crecimiento y no es modificada por el contenido de proteína en el alimento (Ticona, 2009). Los recién nacidos poseen un nivel de creatinina en sangre notablemente alto (Kraft, 2000).

\section{Parámetros sanguíneos de la bilirrubina en llamas}

La bilirrubina se forma a merced de la desintegración de los hematíes viejos y en la medula ósea por la degradación intracorpuscular de la hemoglobina de los eritrocitos inmaduros. Los niveles sanguíneos de bilirrubina total en las diferentes categorías de edad de llamas no presentan diferencias estadísticas (䍚=0.05). Los valores obtenidos están dentro los parámetros reportados por diferentes investigadores.

Los niveles séricos de la bilirrubina en alpacas, no está influenciada por la raza ni el sexo (Garnica, et al., 2003).

\section{Parámetros sanguíneos de glucosa en llamas}

La glucosa es un azúcar simple, su metabolismo oxidativo proporciona la mayor parte de la energía utilizada por el organismo. Los parámetros de glucosa sanguíneos son superiores en llamas DL e inferiores en CD, en llamas DD, BLl son similares a las anteriores (圆=0.05). (Gráfico 8). 


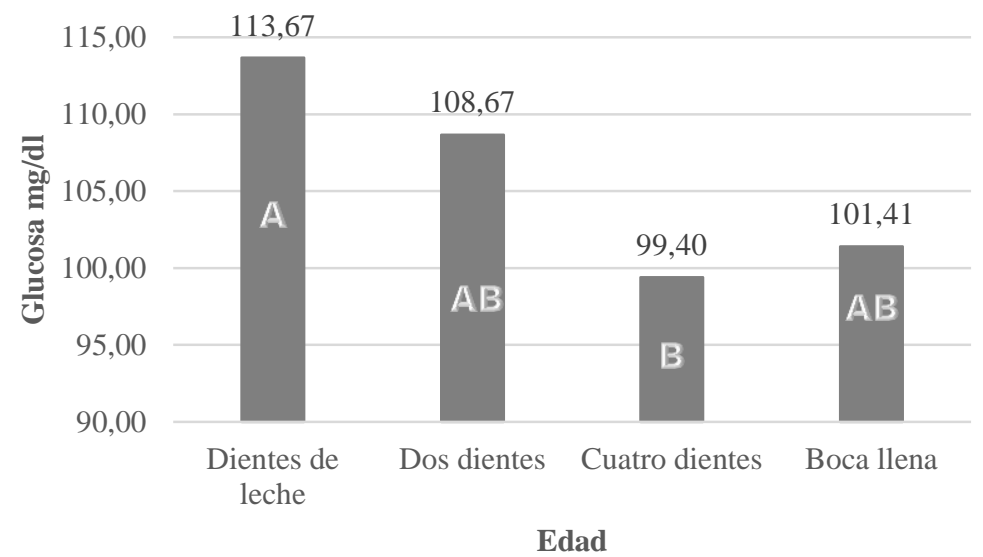

Gráfico 8. Parámetros sanguíneos de glucosa en llamas

Las llamas DL por la intensa actividad física y metabólica presentan niveles sanguíneos fisiológicos superiores de glucosa producto de la absorción, glucogenólisis y gluconeogénesis; a medida que avanza la edad las actividades físicas tienden a disminuir lo que incide a que los niveles de glucosa disminuyan en los animales de mayor edad.

\section{Parámetros sanguíneos de calcio en llamas}

El calcio fisiológicamente es necesario para la contracción muscular, la transmisión del impulso nervioso, la secreción hormonal, la coagulación sanguínea, división, motilidad celular, etc. Los niveles de calcio sanguíneos son superiores en llamas DL e inferiores en $\mathrm{BLl}$, en los animales DD, CD son similares a las anteriores (?=0.05).

En llamas dientes de leche, la intensa actividad metabólica en el crecimiento óseo determina la mayor movilización sanguínea de calcio por esta razón los niveles plasmáticos son superiores. La concentración de calcio disminuye a medida que aumenta la edad debido a la menor movilización; además solo es utilizado para el mantenimiento, el soporte y locomoción del organismo en los animales de mayor edad. (Gráfico 9).

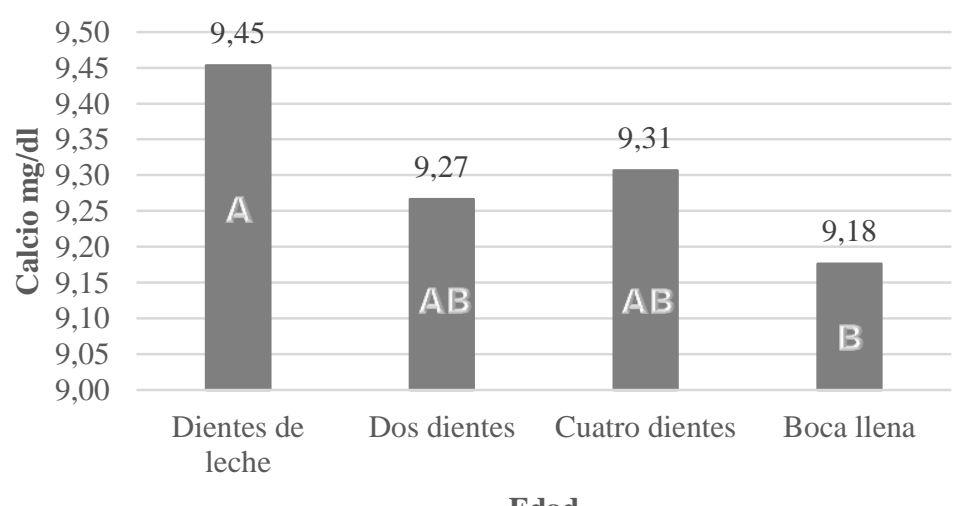

Edad

Gráfico 9. Parámetros sanguíneos de calcio en llamas 
Los valores séricos de calcio en sangre tienden a disminuir con la edad del animal (Doxey, 2000). En animales mayores existe una actividad menor, en machos adultos absorben solo el calcio suficiente para restablecer lo eliminado por orina e intestino (Mc Donald et al., 1995).

\section{Parámetros sanguíneos de fósforo en llamas}

El fosforo es componente de sustancias de gran importancia funcional, tanto desde el punto de vista estructural como metabólico, en el plasma tiene un papel regulador de la absorción. Los niveles sanguíneos de fósforo son superiores en llamas DL e inferiores en DD, CD, BLl que son similares entre sí (可=0.05).

La mayor concentración de fósforo sanguíneo en llamas DL se relacionan al estado fisiológico de crecimiento, desarrollo óseo y muscular que demanda un mayor movimiento de fósforo en sangre; mientras que en llamas de mayor edad la absorción de fósforo es menor y suficientes para cubrir las demandas fisiológicas reproductivas. (Gráfico 10).

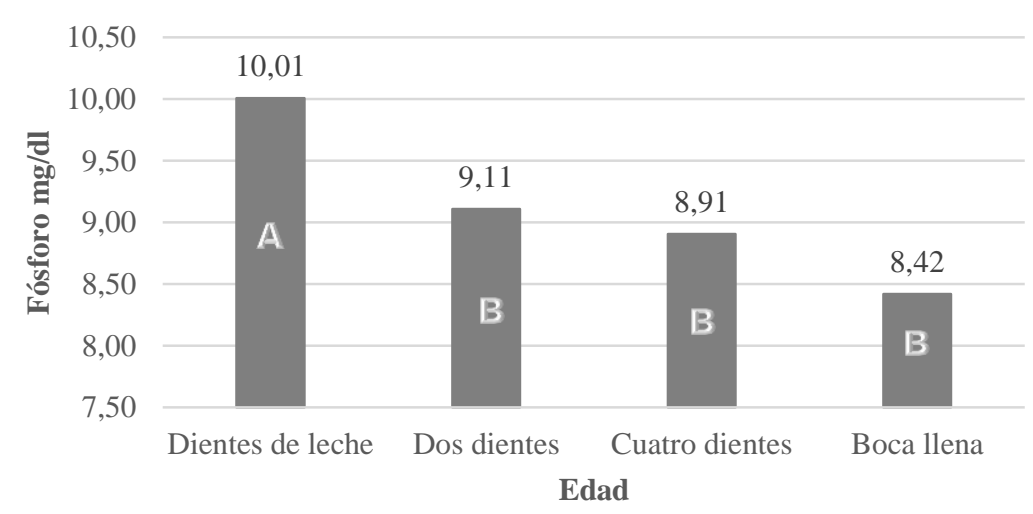

Gráfico 10. Parámetros sanguíneos de fósforo en llamas

El fósforo es absorbido en el intestino delgado por los animales jóvenes en un porcentaje mayor pero no sucediendo de manera similar con los adultos, el porcentaje de absorción en animales jóvenes de un 80 a $100 \%$ y en adultos 50 a $60 \%$ (Blood et al., 1997).

\section{Parámetros sanguíneos del sodio en llamas}

El sodio es el determinante primario de la osmolaridad plasmática y contribuye a la regulación del volumen extracelular. El potasio juega un papel importante en el metabolismo celular, participa en procesos como la síntesis de proteínas y glucógeno, la relación entre sus concentraciones extracelulares e intracelulares es el principal determinante del potencial de reposo de membrana plasmática. Los niveles sanguíneos de sodio y potasio en las diferentes edades de llamas no presentan diferencias estadísticas (? $=0.05$ ). Los valores obtenidos están dentro los parámetros reportados por diferentes investigadores.

\section{CONCLUSIONES}

En su habitad natural las llamas alimentadas en praderas nativas tholar $\mathrm{y}$ pajonal, cada individuo posee patrones diferentes de componentes químicos sanguíneos de proteína total, albúmina, 
globulina, urea nitrógeno, creatinina, glucosa, calcio, fósforo y la actividad enzimática de fosfatasa alcalina y amilasa que pueden cambiar con la edad por la naturaleza de su estado fisiológico. Los niveles de bilirrubina total, sodio, potasio y la actividad enzimática alanina aminotransferasa no varían en las diferentes edades de estos animales.

\section{REFERENCIAS}

Ajata, A. M. (2006). Perfil metabólico de balance de nitrógeno en llamas (Lama glama) alimentado con jipi de quinua y heno de cebada. Tesis para el grado de Ingeniero Zootecnista. Tiwanaku La Paz, Bolivia: Universidad Católica Boliviana San Pablo

Álvarez, C. J.L.; (2008). Bioquímica nutricional y metabólica del bovino el trópico. Primera reimpresión. Editorial Universidad de Antioquia

Benjamín, M. 1991. Manual de patología clínica veterinaria. Noriega Editores. Limusa S.A. de C.V., México

Blood, D.C.; Radostits, O.M.; Arundel, J.H. Gay, C.C. (1997). Medicina Veterinaria Vol II 7 ma edición. Editorial Interamericana, S.A. de C.V., Mc.Graw-Hill. México. D.F

Coaquira, A. K. (2005). Perfil metabólico y peso vivo de llamas (Lama glama) machos alimentados con heno de cebada, alfalfa y kiri de quinua. Tesis para el grado de Ingeniero Zootecnista. Tiwanaku La Paz, Bolivia: Universidad Católica Boliviana San Pablo.

Choque, A. M. E. (2009). Perfil metabólico y evolución de peso vivo de llamas (Lama glama) en jaulas metabólicas en Curahuara de Carangas, departamento Oruro- Bolivia. Tesis para el grado de Ingeniero Zootecnista. Tiwanaku La Paz, Bolivia: Universidad Católica Boliviana San Pablo

Donald MC. P., Edwards, R., Greenhalgh, J. Y Morgan, C. (1999). Nutrición animal. 5ta edición. Editorial Acribia, S.A. Zaragoza España
Doxey, D. L. (2000). Patología clínica y procedimiento de diagnóstico en veterinaria. Editorial El manual moderno. S.A. de C.V. México. D.F

Fowler, M. E. (2010). Medicine and surgery of South American Camelids. Llama, alpaca, vicuña y guanaco. 3ra a edición Iowa University Press. USA. Chapter

Garnica, J., M. Arocutipa Y W. Bravo. (2003). Componentes bioquímicos de la sangre de vicuñas en el altiplano peruano. Resúmenes III Congreso Mundial sobre Camélidos. Potosí. Bolivia

Kraft, W. Y Durr, U. M. (2000). Diagnóstico de laboratorio clínico veterinaria. 4ta edición Editores Médicos, S.A. Gabriela Mistral, Madrid Rafael de Casanova.

Mc Donald, P.; Edwuards, R.; Greenhalgh, J. Y Col. (1995). Nutrición animal. 5ta edición. Editorial Acribia, S.A. Zaragoza España

Quispe, C. Y. S. (2007). Perfil metabólico y ganancia de peso vivo de llamas (Lama glama) alimentadas con tres raciones en época seca; Curahuara de Carangas OruroBolivia. Tesis para el grado de Ingeniero Zootecnista. Tiwanaku La Paz, Bolivia: Universidad Católica Boliviana San Pablo

Radostits, O.M., Gay, C. C., Blood, D.C., Hinchcliff, K. W. (2002). Medicina Veterinaria. 9na edición. Editorial Mc Graw-Hill Interamericana S.A. de C.V. México, D.F

Ticona, B. E. (2009). Concentración de metabolitos y comportamiento de peso vivo en llamas (Lama glama) alimentadas con: paso natural y heno de cebada durante la gestación, postpartum y crías en turco-departamento de Oruro- Bolivia. Tesis para el grado de Ingeniero Zootecnista. Tiwanaku La Paz, Bolivia: Universidad Católica Boliviana San Pablo

Scholz, H. (2001). Control metabólico en ganado usando parámetros en sangre y leche. Memorias XXV Congreso Nacional de Buiatría, Veracruz, México 